\title{
Recent Guidelines and Recommendations for Laboratory Detection of Lupus Anticoagulants
}

\author{
Gary W. Moore, BSc, DBMS, CSci, FIBMS, CBiol, MSB, CertMHS ${ }^{1}$ \\ ${ }^{1}$ Department of Haemostasis and Thrombosis, GSTS Pathology, Guy's \\ \& St. Thomas' NHS Foundation Hospitals Trust, London, England \\ Address for correspondence Gary W. Moore, BSC, DBMS, CSci, FIBMS, \\ CBiol, MSB, CertMHS, Diagnostic Haemostasis \& Thrombosis \\ Laboratories, 4th Floor North Wing, St. Thomas' Hospital, Westminster \\ Semin Thromb Hemost 2014;40:163-171. \\ Bridge Road, London SE1 7EH, England (e-mail: gary.moore@gsts.com).
}

\begin{abstract}
Keywords

- lupus anticoagulants

- guidelines

- dilute Russell viper venom time

- activated partial thromboplastin time

The International Society on Haemostasis and Thrombosis (ISTH) and the British Committee for Standards in Haematology (BCSH) have recently updated their lupus anticoagulant (LA) detection guidelines. The Clinical and Laboratory Standards Institute (CLSI) subsequently published its first LA guideline. General agreement exists on issues such as sample preparation, the use of dilute Russell viper venom time (dRVVT) in diagnostic repertoires, the use of normalized ratios, calculations to demonstrate phospholipid dependence, calculations to demonstrate inhibition, and interpretive reporting. The ISTH recommendation to employ only dRVVT and activated partial thromboplastin time is not mirrored in the BCSH and CLSI documents. The potential for false negatives in mixing tests is acknowledged by all panels, yet they remain mandated by ISTH as there are occasions when they are crucial to diagnostic accuracy. BCSH indicates that a negative mixing test need not exclude the presence of a LA, and CLSI reprioritizes test order to screen-confirm-mix, the latter being considered unnecessary in specific circumstances. Opinions in the guidelines differ on setting cutoff levels (i.e., 97.5th vs. 99th percentile for normally distributed data). All guidelines cover testing of anticoagulated patients, more detail being given by BCSH and CLSI, who suggest that Taipan snake venom time is a useful adjunct test in patients receiving vitamin $\mathrm{K}$ antagonists. Although complete agreement is not apparent, the guidelines represent significant moves toward engendering common practices.
\end{abstract}

Lupus anticoagulants (LA) are somewhat akin to subatomic particles and extrasolar planets, in that they are "detected" by inference, after exclusion of other possible causes of test findings. In the diagnostic setting, this makes LA detection both a challenge and a frustration, and guidelines from various expert groups have been published and updated over recent decades to outline current opinion on best practice. Problems such as antibody heterogeneity, reagent

published online February 5, 2014
Issue Theme Quality in Hemostasis and Thrombosis, Part III; Guest Editors, Emmanuel J. Favaloro, PhD, FFSc (RCPA), Giuseppe Lippi, MD, and Mario Plebani, MD. and analyzer variability, differing interpretation strategies, and absence of gold standards continue to conspire against generation of unequivocally ideal diagnostic strategies. Consequently, it is perhaps unsurprising that incomplete agreement exists between contemporaneous groups and their guidelines, even with some degree of author crossover. Indeed, it has been suggested that consensus guidelines on antiphospholipid antibody testing are particularly prone to
Copyright $\odot 2014$ by Thieme Medical Publishers, Inc., 333 Seventh Avenue, New York, NY 10001, USA. Tel: +1(212) 584-4662.

\section{License terms \\ () (1) $\Theta \circledast$}


biases inherent the process, mainly because they rely heavily on expert opinion. ${ }^{1}$

The International Society on Haemostasis and Thrombosis (ISTH) has updated their guidelines in late $2009^{2}$ (ISTH 2009), and the British Committee for Standards in Haematology (BCSH) has published an update of their previous guidelines in early $2012^{3}$ (BCSH 2012). The Clinical and Laboratory Standards Institute (CLSI) will publish its first LA guideline in $2014^{4}$ (CLSI 2014). In this article, blood samples will be followed on their journey through the convoluted maze of preanalytical variables, testing with different reagents and the myriad issues impacting on interpretation, with reference to main commonalities and contrasts from the above guidelines, which are summarized in - Table 1.

\section{Preanalytical Variables}

Residual platelet material in test plasma can shorten clotting times and mask the presence of LAs, particularly if plasma is frozen/thawed before analysis. ${ }^{5}$ There is full consensus that blood for LA testing, collected into $0.109 \mathrm{~mol} / \mathrm{L}$ trisodium citrate, must be rendered platelet poor by a process of double centrifugation, to achieve a final platelet count of $<10$ $\times 10^{9} / \mathrm{L}$. CLSI 2014 indicates that this may potentially be achieved after the first centrifugation step; however, this approach should be validated by the laboratory, and the second centrifugation step is necessary if the requisite plate- let reduction is not achieved by a single centrifugation. In practice, this will depend on many variables, including centrifugation type and speed, as well as operator technique. Thus, a single centrifugation step may be inefficient for achieving the target of $<10 \times 10^{9} / \mathrm{L}$ in all samples. ${ }^{6}$ The guidelines also differ on the recommended centrifugation technique, with ISTH 2009 recommending differential speeds on first and second spins. BCSH 2012 and CLSI 2014 discourage ultracentrifugation $(>5,000 \mathrm{~g}$ ) in the second step because it may generate microparticles. ${ }^{5,7}$ The previously fashionable filtration through $0.22 \mu \mathrm{m}$ cellulose acetate filters is no longer recommended because it introduces variables such as loss of von Willebrand factor and some other coagulation factors. ${ }^{8}$

Preliminary routine coagulation screening, including prothrombin time, activated partial thromboplastin time (aPTT), and thrombin time, are crucial before LA testing to exclude undiagnosed coagulopathies or undisclosed anticoagulant therapy. However, local regulations may prohibit some laboratories from undertaking testing on patient samples additional to that specifically requested by the clinician. This creates diagnostic challenges and clinicians should be encouraged to order these tests simultaneously to requesting LA testing. CLSI 2014 further recommends employing a LAunresponsive aPTT reagent in routine hemostasis practice to reduce serendipitous findings of LA in asymptomatic patients, and permit interpretation of more sensitive assays unencumbered by the possibility of alternative abnormalities

Table 1 Summary of commonalities and contrasts between recent ISTH, BCSH, and CLSI guidelines for lupus anticoagulant detection

\begin{tabular}{|l|l|l|l|}
\hline Area of recommendation & ISTH 2009 & BCSH 2012 & CLSI 2014 \\
\hline Sample preparation & Double centrifugation & Double centrifugation & Double centrifugation \\
\hline Assays to use & dRVVT and aPTT & dRVVT plus aPTT or others & $\begin{array}{l}\text { dRVVT and aPTT and/or } \\
\text { others }\end{array}$ \\
\hline Testing order & Screen-mix-confirm & Screen-mix-confirm & Screen-confirm-mix \\
\hline Ratio derivation & NPP denominator & NPP denominator & RI mean denominator \\
\hline RI/cutoffs & 99th percentile & 97.5th percentile (if Gaussian) & $\begin{array}{l}\text { 97.5th percentile } \\
\text { (if Gaussian) }\end{array}$ \\
\hline $\begin{array}{l}\text { Calculations for } \\
\text { phospholipid-dependence }\end{array}$ & $\begin{array}{l}\text { \% correction of screen by } \\
\text { confirm, or } \\
\text { LA ratio (screen/confirm) }\end{array}$ & $\begin{array}{l}\text { \% correction of screen by } \\
\text { confirm, or } \\
\text { LA ratio (screen/confirm) }\end{array}$ & $\begin{array}{l}\text { \% correction of screen by } \\
\text { confirm, or } \\
\text { LA ratio (screen/confirm) }\end{array}$ \\
\hline Mixing test & $\begin{array}{l}\text { Perform on 1:1 mixture with NPP; } \\
\text { Interpret with ICA or } \\
\text { mixing test-specific cutoff }\end{array}$ & $\begin{array}{l}\text { Perform on 1:1 mixture } \\
\text { with NPP }\end{array}$ & $\begin{array}{l}\text { Perform on 1:1 mixture } \\
\text { with NPP; } \\
\text { Interpret with ICA or mixing } \\
\text { test-specific cutoff }\end{array}$ \\
\hline Testing patients on VKAs & $\begin{array}{l}\text { Undiluted plasma if INR }<1.5 ; \\
\text { Mix with NPP if INR }>1.5<3.0\end{array}$ & $\begin{array}{l}\text { Screen and confirm on } \\
\text { TSVT + ET or PNP }\end{array}$ & $\begin{array}{l}\text { Screen and confirm on 1:1 } \\
\text { mixture with NPP; } \\
\text { TSVT + ET or PNP }\end{array}$ \\
\hline Testing patients on UFH & $\begin{array}{l}\text { Interpret with caution } \\
\text { Interpretive reporting }\end{array}$ & $\begin{array}{l}\text { Rot recommended } \\
\text { Can detect LA in some } \\
\text { cases where heparin } \\
\text { neutralizer is effective }\end{array}$ \\
\hline
\end{tabular}

Abbreviations: aPTT, activated partial thromboplastin time; BCSH, British Committee for Standards in Haematology; CLSI, Clinical and Laboratory Standards Institute; dRVVT, dilute Russell viper venom time; ET, Ecarin time; ICA, index of circulating anticoagulant; INR, international normalized ratio; ISTH, International Society on Thrombosis and Haemostasis; LA, lupus anticoagulant; NPP, normal pooled plasma; PNP, platelet neutralization procedure; RI, reference interval; TSVT, Taipan snake venom time; UFH, unfractionated heparin; VKA, vitamin K antagonist. 
if coagulation screening is normal. Thus, LA assay data can be interpreted at face value without the need to consider the presence of abnormalities that may mask, mimic, or coexist with LAs.

BCSH 2012, which incorporates clinical management guidelines, recommends that primary thromboprophylaxis is not used for patients with incidentally detected antibodies because thrombosis rates are low, even with persistent antibodies. $^{9-11}$ ISTH 2009 assigns moderate appropriateness to further testing for LA when an elevated routine aPTT is "accidentally" encountered in asymptomatic patients. Interestingly, a recent study revealed that asymptomatic patients positive for all three of LA, anticardiolipin antibodies, and anti- $\beta_{2}$ glycoprotein I antibodies are at high risk of incurring a thromboembolic event, ${ }^{12}$ and it may be that this debate already requires revisiting.

\section{Generating Reference Intervals/Cutoff Levels}

Reagent variability necessitates use of locally derived reference intervals (RIs) specific to the reagent-analyzer pairings in use. ${ }^{13-15}$ In common with many other analytes, RIs for LA assays have historically been derived from the RI mean \pm 2 standard deviations (SD). This is not inappropriate since data from normal donor populations for clotting tests are commonly Gaussian or near Gaussian, or can be made so by data transformation, ${ }^{14,16}$ although this requires confirmation with each assessment to enable data to be suitable for parametric statistics. ${ }^{17}$ The upper limit operates as the cutoff (97.5th percentile) for determining positivity and initiating confirmatory tests, while the RI mean clotting time can be employed to generate normalized ratios.

ISTH 2009 recommends application of the 99th percentile to determine the cutoff, which equates to the RI mean $+2.3 \mathrm{SD}$ for normally distributed data. This introduces potential to reduce the frequency of false-positive results and thereby increase specificity, yet it is a statistical inevitability that this will reduce sensitivity. The recommendation has proven controversial, partly because the guideline states that a 99th percentile value can be derived from a minimum of 40 donors, yet this is not the case and a minimum of 120 has been previously recommended. ${ }^{18} \mathrm{An}$ even greater number of normal individuals is required to generate a 99th percentile value from nonnormally distributed data. BCSH 2012 discusses inaccuracy of RI generation with small sizes, which impinges whether you attempt to calculate 97.5th or 99th percentile. More practical advice is given in the form of validating previously established cutoffs, such as from the reagent manufacturer or from a different analyzer, using just 20 to 60 normal donors. Although accepting that no statistical tool is perfect, CLSI 2014 maintains that generating a RI from its mean \pm 2 SD remains a valid and achievable proposition for diagnostic laboratories. Reference is made to a separate CLSI publication on clinical laboratory RIs, ${ }^{18}$ echoing the BCSH 2012 advice of validating established cutoffs with reduced donor numbers. An important point is made that an elevated LA screening test in isolation is not a false-positive result but merely a prolonged clotting time requiring confirmatory tests to reveal whether or not the initial abnormality is due to a LA. Statistical outliers or other causes of prolonged clotting times will usually generate an equally elevated confirmatory test result for that assay, and crucially, not generate a false-positive interpretation. A false-positive screening test obtained from the use of the 97.5th percentile will not generate false-positive composite interpretation, yet a false-negative resulting from application of the 99th percentile could lead to failure to secure appropriate diagnosis and treatment.

\section{Which Screening Tests to Use and How Many to Use?}

It has long been recognized that no single assay system will detect all LAs and therefore multiple assays, using differing analytical principles, are required to achieve acceptable detection rates. ${ }^{19,20}$ However, ISTH 2009 indicates that the risk of false-positive results is increased to unacceptable levels if more than two screening tests are performed, and restricts assay choice to only dilute Russell viper venom time (dRVVT) for its specificity and aPTT with low phospholipid concentration because of its sensitivity. ISTH 2009 further supports this view according to potential inconsistency between techniques used for additional test methods. Although contentious, these viewpoints are not without logic and warrant further consideration. Aside from the logistics and costs of performing numerous tests on every patient, it is likely that some patients will generate an elevated screening test with at least one test/reagent type, with the chance on this increasing the more tests performed. Such a result could be due to a genuine LA unreactive in other reagents, a "weak" LA, a discrete analytical error or merely because the patient is a natural statistical outlier for that reagent/analyzer pairing as for conventional laboratory testing. Here, it is important to reflect that to identify a cutoff, be it 97.5 th or 99th percentile, some normal individuals ( 2.5 or $1 \%$ for these examples) will be outliers. As different normal individuals may be outliers in different tests, there is an additive effect for "false-positive" identification. As a statistical example, performance of 10 screening tests, with only one needing to be positive for potentially defining positivity for the condition, could theoretically identify $12.5 \%$ of tested (normal) individuals as being positive for the condition using a 97.5th percentile cutoff. In addition, ethnic differences have also been described for dRVVT ratios that can lead to apparent false-positive screening tests. ${ }^{21}$ These non-LA examples, however, would not normally lead to false-positive composite interpretation, for the reasons described above, and the issue becomes more that of performing unnecessary confirmatory tests.

The ISTH 2009 recommendation has its basis in the considerable body of evidence indicating that pairing dRVVT and aPTT achieves good detection rates, which additionally serves to nurture common diagnostic practices. CLSI 2014 recommends dRVVT and LA-responsive aPTT (LAR-aPTT) as the first-line screening assays but does not exclude employing additional tests of different principles during initial analysis or as second-line assays. BCSH 2012 indicates that dRVVT should be one of two tests used in LA screening, the other being an aPTT with proven LA sensitivity, modified aPTT or a 
dilute prothrombin time (dPT). Parity of recommendation for dRVVT is not surprising because this test procedure has repeatedly been shown to be sensitive to $\beta_{2}$ glycoprotein Idependent antibodies and highly correlate with thrombosis. $^{22}$ Familiarity, ease of performance, and cost are likely to ensure aPTT remains the most common partner test for dRVVT. Nevertheless, it is important to recognize that considerable variability in diagnostic performance exists for reagents from different manufacturers for both tests. $^{2,4,5,13,14,17,20}$ In addition, although most commercial dRVVT reagents behave similarly, potentially suggesting common manufacturing practices, occasional deviations in behavior occur. ${ }^{23}$

The different wording to describe "suitable" aPTT reagents is interesting. ISTH 2009 is the only document to specify dilute phospholipid, whereas phrasing in the other two guidelines can encompass routine aPTT reagents without dilute phospholipid but recognized to be (relatively) LAR. Both are potentially fallible. Diluting a phospholipid preparation of LA-unresponsive composition may not perform as well as an undiluted LAR preparation, ${ }^{19,24}$ yet the latter risks missing weaker antibodies if the phospholipid is too concentrated. 22,25 CLSI 2014 deliberately avoids the use of "LAsensitive/LA-insensitive" terminology, based on the concept that "LA sensitivity" is difficult to appropriately define due to the lack of any "gold standard" LA detection method. Finally, although the dRVVT/aPTT pairing cannot guarantee detection of all antibodies, external quality assurance scheme reports continue to validate the pair as an optimal approach sufficient for LA detection/exclusion in most samples assessed. ${ }^{26,27}$

\section{The Case for Other Tests}

That two ${ }^{3,4}$ of three expert guideline panels promote tests other than dRVVT and aPTT warrants further coverage. This stems from not inconsiderable experience of their application in the diagnostic setting and recognition that these assays can detect clinically significant antibodies undetectable in dRVVT and aPTT as well as many that are. ${ }^{28}$

The kaolin clotting time (KCT) is often quoted as being one of the most sensitive assays for LA because of its extremely low phospholipid concentration. ${ }^{5}$ Technical difficulties such as meticulous sample handling, long clotting times, and variation in residual lipid mean that reproducibility is poor relative to other tests and these issues provide the reasons that ISTH 2009 does not recommend this assay. Being unrestricted in length, the CLSI 2014 guideline considers each available assay in more detail, and also covers best practice and limitations. In experienced hands, the KCT is deemed a sensitive LA assay, providing that results are interpreted in light of assay design, which of course holds true for all assays. Bearing in mind that all recent guidelines emphasize the necessity of confirmatory assays, an important limitation for the KCT is that it lacks a widely available confirmatory test, thereby compromising specificity by not fulfilling an indispensable diagnostic criterion. ${ }^{28}$

The silica clotting time (SCT) was first described as a similar test to KCT, with the advantage of being compatible with automated analyzers employing photo-optical clot detection. ISTH 2009 does not recommend kaolin as activator in aPTT-based tests because its particulate nature compromises this detection principle and requires constant mixing to prevent rapid sedimentation. Low-opacity/slow-settling KCT reagents are in fact commercially available, although samplerelated technical difficulties and the lack of confirm test issue persists. SCT was subsequently revised as a paired system with dilute phospholipid in the screen and concentrated phospholipid in the confirm, ${ }^{29}$ ostensibly converting it into an automate-friendly dilute aPTT. This paired system has demonstrated good diagnostic performance when used in conjunction with a dRVVT system. ${ }^{29,30}$ ISTH 2009 also discourages the use of aPTT reagents employing ellagic acid as the contact activator, citing insensitivity to LA. However, inconsistent sensitivity of aPTT reagents to LA is largely due to variation in phospholipid concentration or composition and ellagic acid-containing reagents can be suitable for LA detection. ${ }^{17,25,31}$ Consequently, neither BCSH 2012 nor CLSI 2014 restricts aPTT reagents based on activator alone. The BCSH 2012 recommendation to employ a LAR-aPTT or modified aPTT can reasonably be taken to include use of KCT, SCT, or dilute aPTT.

$\mathrm{dPT}$ is no longer endorsed by ISTH because of the variability in thromboplastin reagents. That premise is particularly true for tissue-derived reagents although LA sensitivity and reagent variability are improved when recombinant reagents are employed. ${ }^{5,22,28}$ Several studies have shown that dPT detects clinically significant antibodies, some unreactive with dRVVT and aPTT. ${ }^{28,32-35}$ A persistent problem has been that different laboratories adopt a variety of modifications with their locally employed thromboplastin, such as the dilutions employed for screen and confirm tests, resulting in significant variability in diagnostic performance. ${ }^{36}$ The relatively recent availability of a standardized commercially available reagent kit has led to the suggestion that LA detection is improved when dRVVT and aPTT are accompanied by dPT. ${ }^{34,37}$

Assays based on snake venoms such as Textarin and Ecarin are not recommended by ISTH 2009 as standardized commercial assays are said to be unavailable. Although this is currently true for Textarin, preparations of Taipan and Ecarin venom for use in LA assays have been available for some years from at least one manufacturer. ${ }^{38}$

The variation in epitope specificities, even for antibodies to domain I of $\beta_{2}$ glycoprotein I, ${ }^{39}$ contributes to the fact that the dRVVT and aPTT combination alone cannot deliver diagnostic certainty. New or alternative assays are commonly evaluated against dRVVT and/or aPTT, and are thus disadvantaged because they may be sensitive to different antibody subpopulations. Comparison against the dRVVT/aPTT combination ostensibly creates a selection bias. The less commonly employed assays can detect LA that will not manifest in dRVVT and aPTT, and at least some of these antibodies are clinically significant. ${ }^{28,32-35,40-43}$ It is impractical and probably unnecessary for all laboratories to adopt extensive LA-assay repertoires, but there is a case for the use of additional assays in select patients or circumstances. ${ }^{3,4,28}$ 


\section{Mixing Tests}

There is a clear continuum of views across the three guidelines concerning mixing tests. ISTH 2009 advocates performing the mixing test immediately after finding an elevated screening test. It is implicit that the confirmatory test is only undertaken if the mixing test reveals inhibition. BCSH 2012 indicates that mixing tests improve specificity, but introduce a dilution factor that may make weak antibodies appear negative. By way of clarification, it states that in the absence of other causes of prolonged clotting times, samples with negative mixing tests but clear positive screen and confirm tests on undiluted plasma can be considered LA positive. ${ }^{44-46}$ It is therefore implicit that all three of the screen, mixing test, and confirmatory test medley are undertaken.

CLSI 2014 goes further and reprioritizes the order of testing to screen, confirm, and mix, the latter only being undertaken if initial testing is not clear cut because its limitations can lead to false-negative reporting. ${ }^{5,20,22,28,38,44-50}$ Omitting the mixing test is recommended only where there is no evidence of other causes of elevated clotting times, which can be obtained in many cases from the CLSI 2014 recommendation of performing a routine coagulation screen that includes a LA-unresponsive aPTT. In addition, the confirmatory test (on undiluted plasma) must not only mathematically correct an elevated screening test result but should also return into the RI. ${ }^{51}$ Otherwise, the elevated results could derive from an abnormality undetected in coagulation screen, or be a LA sufficiently potent to achieve some degree of resistance to the swamping effect of confirm reagent. In such circumstances, the mixing test limitation can be advantageous if the dilution reduces the antibody's effect and facilitates overwhelming it with the confirm reagent. Where a coexisting abnormality is suspected, performing screen and confirm tests on 1:1 mixtures with normal pooled plasma (NPP) to correct it can reveal the presence of a LA. All guidelines agree that mixing tests should be performed on a $1: 1$ mixture of test plasma and NPP.

No guideline before ISTH 2009 gave definitive advice for interpreting mixing tests, so it was especially welcome that this publication fully addresses the issue. Two options are given. One entails using a mixing test-specific RI, which will be narrower than that for undiluted plasma, and thus have a lower cutoff due to clotting times of normal samples at the extremes being compensated upon mixing with NPP. ${ }^{22,46}$ The lower cutoff increases sensitivity in comparison to assessing against the cutoff for undiluted plasma. The alternative is application of the index of circulating anticoagulant (ICA) $)^{52}$ as shown below:

$$
\mathrm{ICA}(\%)=\frac{\text { Screen } 1: 1 \mathrm{mix}(\mathrm{s})-\text { Screen NPP }(\mathrm{s})}{\text { Screen undiluted test plasma }(\mathrm{s})} \times 100
$$

The ICA is advantageous because it assesses test plasma clotting times relative to the NPP in which it is diluted. A mixing test-specific cutoff can do so if applied to ratios and not to clotting times. CLSI 2014 concurs with these recommendations, whereas BCSH 2012 does not cover the issue. However, no mathematical method for assessing mixing test data can perfectly discriminate between factor deficiencies and inhibitors, and cutoffs vary between test types and reagents. ${ }^{53,54}$

\section{Ratios}

It is common practice to convert clotting times for screen and confirm tests to normalized ratios via NPP values. This improves inter- and intra-assay variation by mitigating differences in operator and/or analyzer performance, reagent quality and stability issues, and NPP clotting time variation with different reagents. ${ }^{13,14,55}$ Ratios also compensate for the fact that NPP clotting times with paired screen and confirm reagents can differ. ${ }^{20} \mathrm{BCSH}$ has recommended this practice since $1991^{3,20,56}$ and it is adopted by ISTH 2009.

The CLSI 2014 approach differs, in that the recommendation is to normalize against the RI mean clotting time and not against the NPP value. The rationale here is that not all NPPs, or batches of the same NPP, generate the same clotting times with different reagents for the same test type, ${ }^{13,14,55,57}$ so that false-positive and false-negative results can ensue if an NPP value is toward or beyond an extreme of a RI. ${ }^{55}$ Establishing the RI and its mean by analyzing normal donors over several days accounts for innate technique variability, ${ }^{18}$ and running an NPP as a normal control will identify sudden analytical difficulties. The use of a mean RI value also simplifies the process of automated calculations, for example, in laboratory information systems.

\section{Confirmatory Tests}

All three guidelines agree that confirmatory tests used to demonstrate phospholipid dependence must be based on the screening test(s) that was abnormal. It is not explicitly stated in ISTH 2009, yet this is an update of ISTH $1995^{19}$ where it is a clear recommendation and thus remains current. This is a crucial point, as some laboratories apply only the mixing test in response to an elevated aPTT and are at risk of both loss of specificity and increased false-negative interpretations unless dRVVT testing is also positive. ${ }^{44-49,57,58}$

ISTH 2009 indicates that confirmatory tests must be performed by increasing the phospholipid concentration, with bilayer or hexagonal (II) phase phospholipid. BCSH 2012 does not specify the type, and suggests employing high phospholipid concentration, platelet neutralization procedure (PNP), or LA-insensitive reagent. ISTH 2009 advises against freeze/thawed platelets because of reagent batch inconsistencies, which perhaps should have specified that this relates particularly to locally prepared reagents. ${ }^{59}$ All confirmatory tests are versions of their parent screening test rendered LA unresponsive and the specific reference to such reagents in BCSH 2012 alludes to techniques pairing LAR and LA-unresponsive aPTT reagents. CLSI 2014 gives details of recommended available confirmatory tests for each screening test, including considerations of limitations, and ostensibly concurs.

Another welcome first for ISTH is a recommended calculation to demonstrate or exclude phospholipid dependence, 
the percentage correction of screen by confirm, as shown below for ratios:

Percentage correction of ratio $=\frac{\text { Screen ratio }- \text { Confirm ratio }}{\text { Screen ratio }} \times 100$

BCSH has championed this calculation since 1991, with a correction of $10 \%$ or more being indicative of the presence of a LA, whereas ISTH 2009 indicates that the cutoff should be locally derived. CLSI 2014 offers alternatives specific to particular assays. The hexagonal phase phospholipid neutralization test and PNP confirmatory test commonly used for aPTT are assessed using deltas, ${ }^{60,61}$ while the normalized screen to confirmatory ratio, as below, is recommended for dRVVT, SCT, and dPT when used with paired screen and confirm reagents. BCSH 2012 suggests that this as an alternative to percentage correction of ratio, and CLSI 2014 suggests the latter as another option for dRVVT.

$$
\text { Normalized screen } \text { confirm ratio }=\frac{\text { Screen ratio }}{\text { Confirm ratio }}
$$

\section{Integrated Testing}

Integrated testing involves performing screen and confirm assays (i.e., low and high phospholipid concentration) on every patient, and then mathematically assessing for correction irrespective of whether the screen is elevated beyond a predetermined cutoff. $^{29,50,51,62,63}$ Detection of weaker LAs is a particular advantage of this approach, where the prolongation of a patient's basal clotting time is insufficient to exceed the upper reference limit of a screening test, yet the screen and confirm discrepancy reveals the antibody. ${ }^{49,64,65}$ Some integrated tests are performed on a 1:1 mixture with NPP to simultaneously correct factor deficiencies and demonstrate inhibition. However, they inevitably risk diluting some antibodies such that even screen and confirm discordance does not manifest because the dilution compromises screening test elevation such that a significant difference is not apparent. ${ }^{49,65}$ CLSI 2014 reserves its definition of integrated tests for those that incorporate dilution in NPP, while ISTH 2009 includes those performed on undiluted test plasma and a separate mixing test. The specifics are not covered in BCSH 2012.

\section{The Integrated Test and Mixing Study Debate}

A statement in ISTH 2009 that performing screen and confirm tests in parallel on undiluted plasma does not, in principle, require subsequent performance of a mixing test and has prompted useful and revealing debate. ${ }^{48,49,51,62,63,66}$ The principle arises from the fact that the traditional testing order of screen-mix-confirm is circumvented and the presence of a LA can be immediately apparent without performing the mixing test. Some have interpreted that statement to mean that mixing tests are no longer mandated by ISTH if integrated testing is undertaken and expressed concerns, ${ }^{48,63}$ resulting in clarification that this was not the intended message. $^{66}$

LAs with a degree of resistance against the "overwhelming" effect of confirmatory reagents can lead to a confirm result above the confirmatory test RI. Many of these will nonetheless have generated a screen result sufficiently higher than the confirm to achieve a positive interpretation. ${ }^{48,51}$ However, the possibility of a coexisting or alternative abnormality must be considered, and performance of both screen and confirm on 1:1 mixtures with NPP can aid discriminating between potent LA, LA + coexisting abnormality, or non-LA abnormality. ${ }^{20,38,51}$ There are occasions when screen and confirm for a genuine LA are elevated to a similar extent such that integrated testing on undiluted plasma generates a false-negative interpretation, implying a nonphospholipiddependent abnormality. Again, screen and confirm mixing tests can be invaluable in this scenario by reducing the antibody's effects and revealing a screen and confirm discrepancy. ${ }^{48,51,62}$

Although considered rare, the LA cofactor effect cannot, by definition, be demonstrated without mixing tests, and integrated testing alone will not detect this phenomenon. ${ }^{62,66}$ Personal experience suggests that these LAs are usually evident in undiluted plasma, and if not clear cut, will receive mixing tests anyway. Such samples can be problematic for those laboratories that do not perform mixing studies. ${ }^{67}$

It has also been demonstrated that test and reagent type can influence the diagnostic efficacy of mixing tests. ${ }^{63,68}$ Assays with higher specificity are less likely to require mixing tests to clearly demonstrate the presence of a LA.

The CLSI 2014 recommendations for when mixing tests can be omitted or not, crystallize this debate, and they affirm that the decision can only be made on a case-by-case basis. It is not a choice of performing mixing tests on everyone or no one, it is a case of scrutinizing raw data to see if mixing tests will tell you anything you do not already know.

\section{Testing Anticoagulated Patients}

All three guidelines concur that testing for LAs in patients receiving vitamin $\mathrm{K}$ antagonists (VKA) and therapeutic doses of unfractionated heparin (UFH) should be postponed until anticoagulation has been discontinued for a suitable period of time. BCSH 2012 indicates that brief discontinuation of VKA therapy is not a high-risk strategy in most cases, where decisions on advisability of long-term anticoagulation are required. Bridging with low-molecular-weight heparin (LMWH) can be employed. However, the reality for diagnostic laboratories is that they are often asked to test for LAs in anticoagulated patients, often without knowing a patient is receiving them until laboratory results suggest so, and guidance is required.

\section{Vitamin K Antagonists}

ISTH 2009 indicates that dRVVT and aPTT can be performed on undiluted plasma if the international normalized ratio (INR) is less than 1.5. If the INR is between 1.5 and 3.0, undertaking the tests on 1:1 mixtures with NPP can be considered, with recognition of the dilution effect. Integrated tests and procedures employing "VKA-insensitive" snake venoms are not recommended as it is suggested that they require further critical evaluation. 
BCSH 2012 and CLSI 2014 advocate performing screen and confirm tests on 1:1 mixtures with NPP but do not restrict this practice to plasmas with INRs $\leq 3.0$. Careful data interpretation of mixing tests has been shown to reveal LAs in some patients with INRs $>3.0 .^{38}$ BCSH 2012 states that an elevated screen on the mixture can be taken as grounds to suspect an inhibitor and the confirm will demonstrate phospholipid dependence. Both documents make an important point that negative testing in mixing studies on these patients does not exclude the presence of a LA because of the dilution effect. CLSI 2014 gives worked examples of a probable negative and definite positive set of results. Testing undiluted plasma can lead to false-positive or false-negative results, and is thus not recommended, ${ }^{13,17,20,69}$ although some workers contend that it can be reliable. ${ }^{70,71}$ Both documents suggest that Taipan snake venom time (TSVT) can be a useful secondary screening test for patients on VKAs, accompanied by Ecarin time (ET) or a PNP as confirmatory test. ${ }^{20,28,33,38,41,72,73}$

\section{Heparins}

Both ISTH 2009 and CLSI 2014 urge caution when interpreting results on patients receiving UFH, while BCSH 2012 specifies that LA testing should not be undertaken on patients receiving therapeutic doses as it may cause erroneous results. CLSI 2014 discusses reagent-integral heparin neutralizers (as found in most commercial dRVVT reagents) in more detail, indicating that they quench heparin only up to a specified level, and it must be demonstrated that this has occurred before result interpretation is undertaken. It also discusses heparin-neutralizing compounds that are added to plasma before analysis and gives worked examples of unquenched UFH and a genuine LA after full UFH neutralization. BCSH 2012 and CLSI 2014 indicate the possibility of false-positive interpretations when a PNP is used as a confirmatory test. LMWH has less effect on LA assays, particularly dRVVT reagents, as the heparin neutralizers are usually capable of covering prophylactic doses.

\section{New Anticoagulants}

Only CLSI 2014 gives evidenced detail of the potential interferences by the newer anticoagulants, information on which is still emerging. Direct thrombin inhibitors (DTI), such as dabigatran and argatroban, interfere with all LA assays and introduce a significant risk of falsepositive results. ${ }^{17,74}$ Although the multiple acquired factor deficiency of VKA anticoagulation can often be corrected in a mixing test and reveal a LA, there is no such correction with DTIs or factor Xa (FXa) inhibitors as there is usually no underlying factor deficiency. ${ }^{17}$ The FXa inhibitor rivaroxaban interferes with dRVVT analysis for LA more so than aPTT and has a variable, reagent-dependent effect on prothrombin time-based assays. ${ }^{17,75-77}$ TSVT coupled with ET has been shown to be a sensitive test for detecting LAs in patients receiving rivaroxaban because both venom fractions are prothrombin activators and thus unaffected by FXa inhibition. ${ }^{76}$

\section{Reporting}

All three guidelines state that the numerical results should be accompanied by an interpretation indicating whether the findings are consistent with the presence or absence of an LA (or more correctly the inability to detect an LA). ISTH 2009 discourages use of comments such as "borderline" while CLSI 2014 suggests that the use of "indeterminate" can be useful where a panel of assay results does not permit clear distinction between LA detection or lack thereof. Each document supports the recommendation to retest in no less than 12 weeks when a LA is initially detected.

\section{Conclusion}

Commonalities between the three guidelines show that we have come a long way toward wider understanding, consensus, and common practices since guidelines were first published over two decades ago. Few would argue against inclusion of dRVVT in any diagnostic repertoire and we have in depth understanding of performance and limitations of all tests available to us. The ISTH 2009 recommendation to employ only dRVVT and aPTT is not surprising since there is a wealth of evidence, unlikely to be surpassed, that this pairing constitutes a sensitive and specific diagnostic strategy. BCSH 2012 and CLSI 2014 consider that there is sufficient evidence for other tests that they should not be discounted in their entirety. Proponents of these assays support them on the basis of published evidence and direct clinical experience. ${ }^{1}$ There is more debate to be had about the place of mixing tests in the LA analytical hierarchy, although it does seem that they can be omitted in certain circumstances. The immediate future probably holds little more than refinements of what we already have until such time as the pathological antibodies causing the in vitro LA phenomenon have been fully characterized. If and when those assays are available to diagnostic laboratories, the days of challenging, fascinating, and frustrating searches for ghosts may be done.

\section{Acknowledgment}

The author thanks Marlies Ledford-Kraemer for reviewing the article and her insightful comments and suggestions.

\section{References}

1 Wong RCW, Favaloro EJ. A consensus approach to the formulation of guidelines for laboratory testing and reporting of antiphospholipid antibody assays. Semin Thromb Hemost 2008;34(4):361-372

2 Pengo V, Tripodi A, Reber G, et al; Subcommittee on Lupus Anticoagulant/Antiphospholipid Antibody of the Scientific and Standardisation Committee of the International Society on Thrombosis and Haemostasis. Update of the guidelines for lupus anticoagulant detection. J Thromb Haemost 2009;7(10):1737-1740

3 Keeling D, Mackie I, Moore GW, Greer IA, Greaves M; British Committee for Standards in Haematology. Guidelines on the investigation and management of antiphospholipid syndrome. Br J Haematol 2012;157(1):47-58 
4 CLSI. Laboratory Testing for the Lupus Anticoagulant; Approved Guideline. CLSI document H60-A. Wayne, PA: Clinical and Laboratory Standards Institute; 2014

5 Devreese K, Hoylaerts MF. Laboratory diagnosis of the antiphospholipid syndrome: a plethora of obstacles to overcome. Eur J Haematol 2009;83(1):1-16

6 Lippi G, Rossi R, Ippolito L, et al. Influence of residual platelet count on routine coagulation, factor VIII and factor IX testing in post freeze-thaw samples. Semin Thromb Hemost 2013;39(7):834-839

7 Sletnes KE, Gravem K, Wisløff F. Preparation of plasma for the detection of lupus anticoagulants and antiphospholipid antibodies. Thromb Res 1992;66(1):43-53

8 Favaloro EJ. Preanalytical variables in coagulation testing. Blood Coagul Fibrinolysis 2007;18(1):86-89

9 Girón-González JA, García del Río E, Rodríguez C, RodríguezMartorell J, Serrano A. Antiphospholipid syndrome and asymptomatic carriers of antiphospholipid antibody: prospective analysis of 404 individuals. J Rheumatol 2004;31(8):1560-1567

10 de Groot PG, Lutters B, Derksen RH, Lisman T, Meijers JC, Rosendaal FR. Lupus anticoagulants and the risk of a first episode of deep venous thrombosis. J Thromb Haemost 2005;3(9): 1993-1997

11 Metjian A, Lim W. ASH evidence-based guidelines: should asymptomatic patients with antiphospholipid antibodies receive primary prophylaxis to prevent thrombosis? Hematology (Am Soc Hematol Educ Program) 2009:247-249

12 Pengo V, Ruffatti A, Legnani C, et al. Incidence of a first thromboembolic event in asymptomatic carriers of high-risk antiphospholipid antibody profile: a multicenter prospective study. Blood 2011;118(17):4714-4718

13 Lawrie AS, Mackie IJ, Purdy G, Machin SJ. The sensitivity and specificity of commercial reagents for the detection of lupus anticoagulant show marked differences in performance between photo-optical and mechanical coagulometers. Thromb Haemost 1999;81(5):758-762

14 Gardiner C, Mackie IJ, Malia RG, et al. The importance of locally derived reference ranges and standardized calculation of dilute Russell's viper venom time results in screening for lupus anticoagulant. Br J Haematol 2000;111(4):1230-1235

15 Miyakis S, Lockshin MD, Atsumi T, et al. International consensus statement on an update of the classification criteria for definite antiphospholipid syndrome (APS). J Thromb Haemost 2006;4(2): 295-306

16 Gerbutavicius R, Fareed J, Messmore HLJr, et al. Reference intervals of the dilute tissue thromboplastin inhibition and dilute Russell's viper venom tests revisited. Clin Appl Thromb Hemost 2002;8(2): 115-124

17 Kershaw G, Suresh S, Orellana D, Nguy YM. Laboratory identification of lupus anticoagulants. Semin Thromb Hemost 2012;38(4): 375-384

18 CLSI. Defining, Establishing, and Verifying Reference Intervals in the Clinical Laboratory; Approved Guideline-Third Edition. CLSI document EP28-A3c. Wayne, PA: Clinical and Laboratory Standards Institute; 2008

19 Brandt JT, Triplett DA, Alving B, Scharrer I. Criteria for the diagnosis of lupus anticoagulants: an update. On behalf of the Subcommittee on Lupus Anticoagulant/Antiphospholipid Antibody of the Scientific and Standardisation Committee of the ISTH. Thromb Haemost 1995;74(4):1185-1190

20 Greaves M, Cohen H, Machin SJ, Mackie I. Guidelines on the investigation and management of the antiphospholipid syndrome. Br J Haematol 2000;109(4):704-715

21 Jerrard-Dunne P, Evans A, McGovern R, et al. Ethnic differences in markers of thrombophilia: implications for the investigation of ischemic stroke in multiethnic populations: the South London Ethnicity and Stroke Study. Stroke 2003;34(8):1821-1826

22 Tripodi A. Laboratory testing for lupus anticoagulants: a review of issues affecting results. Clin Chem 2007;53(9):1629-1635
23 McGlasson DL, Fritsma GA. Comparison of six dilute russell viper venom time lupus anticoagulant screen/confirm assay kits. Semin Thromb Hemost 2013;39(3):315-319

24 Blanco AN, Grand BE, Pieroni G, Peñalva LB, Voto LS, Lazzari MA. Behavior of diluted activated partial thromboplastin time in pregnant women with a lupus anticoagulant. Am J Clin Pathol 1993;100(2):99-102

25 Kumano O, Ieko M, Naito S, Yoshida M, Takahashi N. APTT reagent with ellagic acid as activator shows adequate lupus anticoagulant sensitivity in comparison to silica-based reagent. J Thromb Haemost 2012;10:2338-2343

26 Moffat KA, Ledford-Kraemer MR, Plumhoff EA, et al. Are laboratories following published recommendations for lupus anticoagulant testing? An international evaluation of practices. Thromb Haemost 2009;101(1):178-184

27 Jennings I, Kitchen S, Kitchen DP, Woods TA, Walker ID. ISTH/SSC lupus anticoagulant testing guidelines: how far have these been adopted by laboratories? J Thromb Haemost 2011;9(10): 2117-2119

28 Moore GW. Clinical utility of the less commonly employed assays for lupus anticoagulation detection: the evidence. J Coag Dis 2010; 2:69-79

29 Chantarangkul V, Tripodi A, Arbini A, Mannucci PM. Silica clotting time (SCT) as a screening and confirmatory test for detection of the lupus anticoagulants. Thromb Res 1992;67(4):355-365

30 Norbis F, Barbui T, Galli M. Diluted Russell's viper venom time and colloidal silica clotting time for the identification of the phospholipid-dependent inhibitors of coagulation. Thromb Res 1997; 85(5):427-431

31 Jacobsen EM, Barna-Cler L, Taylor JM, Triplett DA, Wisløff F. The Lupus Ratio test-an interlaboratory study on the detection of Lupus anticoagulants by an APTT-based, integrated, and semiquantitative test. Fifth International Survey of Lupus Anticoagulants-ISLA 5. Thromb Haemost 2000;83(5):704-708

32 Liestøl S, Jacobsen EM, Wisløff F. Dilute prothrombin time-based lupus ratio test. Integrated LA testing with recombinant tissue thromboplastin. Thromb Res 2002;105(2):177-182

33 Moore GW, Smith MP, Patel Y, Savidge GF. The Activated Seven Lupus Anticoagulant (ASLA) assay: a new test for lupus anticoagulants (LAs). Evidence that some LAs are detectable only in extrinsic pathway-based assays. Blood Coagul Fibrinolysis 2002; 13(3):261-269

34 Mackie IJ, Lawrie AS, Greenfield RS, Guinto ER, Machin SJ. A new lupus anticoagulant test based on dilute prothrombin time. Thromb Res 2004;114:673-674 Abstract TH25

35 Devreese KMJ. Evaluation of a new commercial dilute prothrombin time in the diagnosis of lupus anticoagulants. Thromb Res 2008; 123(2):404-411

36 Jennings I, Mackie I, Arnout J, Preston FE; UK National External Quality Assessment Scheme for Blood Coagulation. Lupus anticoagulant testing using plasma spiked with monoclonal antibodies: performance in the UK NEQAS proficiency testing programme. J Thromb Haemost 2004;2(12):2178-2184

37 Lawrie AS, Mackie IJ, Purdy G, Greenfield RS, Guinto ER, Machin SJ Lupus anticoagulant testing using a dilute prothrombin time with confirm procedure. J Thromb Haemost 2005;3(Suppl 1):Abstract P1817

38 Moore GW. Combining Taipan snake venom time/Ecarin time screening with the mixing studies of conventional assays increases detection rates of lupus anticoagulants in orally anticoagulated patients. Thromb J 2007;5:12

39 de Groot PG, Urbanus RT. The future of antiphospholipid antibody testing. Semin Thromb Hemost 2012;38(4):412-420

40 Forastiero RR, Cerrato GS, Carreras LO. Evaluation of recently described tests for detection of the lupus anticoagulant. Thromb Haemost 1994;72(5):728-733

41 Moore GW, Smith MP, Savidge GF. The Ecarin time is an improved confirmatory test for the Taipan snake venom time in warfarinized 
patients with lupus anticoagulants. Blood Coagul Fibrinolysis 2003;14(3):307-312

42 Galli M, Borrelli G, Jacobsen EM, et al. Clinical significance of different antiphospholipid antibodies in the WAPS (warfarin in the antiphospholipid syndrome) study. Blood 2007;110(4): $1178-1183$

43 Moore GW, Rangarajan S, Savidge GF. The activated seven lupus anticoagulant assay detects clinically significant antibodies. Clin Appl Thromb Hemost 2008;14(3):332-337

44 Male C, Lechner K, Speiser W, Pabinger I. Transient lupus anticoagulants in children: stepwise disappearance of diagnostic features. Thromb Haemost 2000;83(1):174-175

45 Thom J, Ivey L, Eikelboom J. Normal plasma mixing studies in the laboratory diagnosis of lupus anticoagulant. J Thromb Haemost 2003;1(12):2689-2691

46 Moore GW, Savidge GF. The dilution effect of equal volume mixing studies compromises confirmation of inhibition by lupus anticoagulants even when mixture specific reference ranges are applied. Thromb Res 2006;118(4):523-528

47 Arnout J. Antiphospholipid syndrome: diagnostic aspects of lupus anticoagulants. Thromb Haemost 2001;86(1):83-91

48 Favaloro EJ, Bonar R, Zebeljan D, Kershaw G, Marsden K. Laboratory investigation of lupus anticoagulants: mixing studies are sometimes required. J Thromb Haemost 2010;8(12):2828-2831

49 Reber G, Meijer P. In ECAT veritas? Lupus 2012;21(7):722-724

50 Aboud M, Roddie C, Ward C, Coyle L. To mix with pooled normal plasma or not to mix: a comparative study of 2 approaches for assessing lupus anticoagulant inhibitory activity in the dilute Russell viper venom method. Clin Chem 2007;53(1):143-145

51 Devreese KMJ. No more mixing tests required for integrated assay systems in the laboratory diagnosis of lupus anticoagulants? J Thromb Haemost 2010;8(5):1120-1122

52 Rosner E, Pauzner R, Lusky A, Modan M, Many A. Detection and quantitative evaluation of lupus circulating anticoagulant activity. Thromb Haemost 1987;57(2):144-147

53 Kershaw G, Orellana D. Mixing tests: diagnostic aides in the investigation of prolonged prothrombin times and activated partial thromboplastin times. Semin Thromb Hemost 2013;39(3):283-290

54 Kumano O, Ieko M, Naito S, Yoshida M, Takahashi N, Takashi A. Index of circulating anticoagulant cut-off value establishment in activated partial thromboplastin time mixing test for lupus anticoagulant diagnosis. J Thromb Haemost 2013;11(10):1919-1922

55 Moore GW, Brown KL, Bromidge ES, Drew AJ, Ledford-Kraemer MR. Lupus anticoagulant detection: out of control? Int J Lab Hematol 2013;35(2):128-136

56 Machin SJ, Giddings JC, Greaves M, et al. Guidelines on testing for the lupus anticoagulant. Lupus Anticoagulant Working Party on behalf of the BCSH Haemostasis and Thrombosis Task Force. J Clin Pathol 1991;44(11):885-889

57 Jennings I, Greaves M, Mackie IJ, Kitchen S, Woods TA, Preston FE; UK National External Quality Assessment Scheme for Blood Coagulation. Lupus anticoagulant testing: improvements in performance in a UK NEQAS proficiency testing exercise after dissemination of national guidelines on laboratory methods. $\mathrm{Br} \mathrm{J}$ Haematol 2002;119(2):364-369

58 Dembitzer FR, Ledford Kraemer MR, Meijer P, Peerschke El. Lupus anticoagulant testing: performance and practices by north american clinical laboratories. Am J Clin Pathol 2010;134(5):764-773

59 Cappucci G, Grandone E, Giuliani N, Margaglione M, Di Minno G. The use of frozen-thawed platelet-derived phospholipids as a confirmatory test for the diagnosis of lupus anticoagulants. Comparison with two commercial confirmatory system tests. Thromb Res 1999;94(6):373-380
60 Triplett DA, Barna LK, Unger GA. A hexagonal (II) phase phospholipid neutralization assay for lupus anticoagulant identification. Thromb Haemost 1993;70(5):787-793

61 Reber G, Roisin JP, Migaud M, de Moerloose P. Comparison of a platelet neutralization procedure with a hexagonal phase phospholipid neutralization assay as confirmatory tests for the presence of lupus anticoagulants. Thromb Res 1994;73(2): 131-135

62 Tripodi A. To mix or not to mix in lupus anticoagulant testing? That is the question. Semin Thromb Hemost 2012;38(4):385-389

63 Hong SK, Hwang SM, Kim JE, Kim HK. Clinical significance of the mixing test in laboratory diagnoses of lupus anticoagulant: the fate of the mixing test in integrated lupus anticoagulant test systems. Blood Coagul Fibrinolysis 2012;23(8):739-744

64 Jacobsen EM, Wisløff $F$. False negative screening tests for lupus anticoagulants-an unrecognized problem? Thromb Res 1996; 82(5):445-451

65 Moore GW, Henley A, Greenwood CK, Rangarajan S. Further evidence of false negative screening for lupus anticoagulants. Thromb Res 2008;121(4):477-484

66 Tripodi A, Pengo V. More on: laboratory investigation of lupus anticoagulants: mixing studies are sometimes required. J Thromb Haemost 2011;9(10):2126-2127

67 Favaloro EJ, Bonar R, Marsden K. Internal quality control and external quality assurance in testing for antiphospholipid antibodies: part II-lupus anticoagulant. Semin Thromb Hemost 2012; 38(4):404-411

68 Chantarangkul V, Biguzzi E, Asti D, Palmucci C, Tripodi A. Laboratory diagnostic outcome applying detection criteria recommended by the Scientific and Standardization Committee of the ISTH on Lupus Anticoagulant. Thromb Haemost 2013;110(1): 46-52

69 Jouhikainen T. Detection of lupus anticoagulant by means of dilute Russell's viper venom time is affected by oral anticoagulant therapy. Blood Coagul Fibrinolysis 1990;1(6):627-632

70 Olteanu H, Downes KA, Patel J, Praprotnik D, Sarode R. Warfarin does not interfere with lupus anticoagulant detection by dilute Russell's viper venom time. Clin Lab 2009;55(3-4):138-142

71 Tripodi A, Chantarangkul V, Clerici M, Mannucci PM. Laboratory diagnosis of lupus anticoagulants for patients on oral anticoagulant treatment. Performance of dilute Russell viper venom test and silica clotting time in comparison with Staclot LA. Thromb Haemost 2002;88(4):583-586

72 Rooney AM, McNally T, Mackie IJ, Machin SJ. The Taipan snake venom time: a new test for lupus anticoagulant. J Clin Pathol 1994; 47(6):497-501

73 Parmar K, Lefkou E, Doughty H, Connor P, Hunt BJ. The utility of the Taipan snake venom assay in assessing lupus anticoagulant status in individuals receiving or not receiving an oral vitamin K antagonist. Blood Coagul Fibrinolysis 2009;20(4):271-275

74 Halbmayer WM, Weigel G, Quehenberger P, et al. Interference of the new oral anticoagulant dabigatran with frequently used coagulation tests. Clin Chem Lab Med 2012;50(9):1601-1605

75 Merriman E, Kaplan Z, Butler J, Malan E, Gan E, Tran H. Rivaroxaban and false positive lupus anticoagulant testing. Thromb Haemost 2011;105(2):385-386

76 van Os GM, de Laat B, Kamphuisen PW, Meijers JC, de Groot PG. Detection of lupus anticoagulant in the presence of rivaroxaban using Taipan snake venom time. J Thromb Haemost 2011;9(8): 1657-1659

77 van Veen JJ, Smith J, Kitchen S, Makris M. Normal prothrombin time in the presence of therapeutic levels of rivaroxaban. $\mathrm{Br} \mathrm{J}$ Haematol 2013;160(6):859-861 